\title{
Review
}

Journal of Innate
Immunity

J Innate Immun 2013;5:304-314

Received: November 1, 2012

DOI: $10.1159 / 000350282$

Accepted after revision: February 27, 2013

Published online: April 6, 2013

\section{Regulation of Circulating Neutrophil Numbers under Homeostasis and in Disease}

\author{
Natasha Strydom Sara M. Rankin \\ Leukocyte Biology Section, National Heart and Lung Institute, Faculty of Medicine, Imperial College London, \\ London, UK
}

\section{Key Words}

Neutrophils · Bone marrow · Mobilization · Clearance ·

CXCR4 · CXCR2 · CXCL12 - Chemokines

\begin{abstract}
Neutrophils are the most abundant circulating leukocyte and play a fundamental role in the innate immune response. Patients with neutropenia, leukocyte adhesion deficiency syndrome or chronic granulomatous disease are particularly prone to bacterial and fungal infection. However, the highly destructive capacity of these cells also increases the potential for neutrophil damage to healthy tissues, as seen in a number of inflammatory diseases such as rheumatoid arthritis and chronic obstructive pulmonary disease. The homeostatic control of circulating neutrophil levels is thus critical, as an imbalance can result in overwhelming infection or inappropriate inflammatory states. Neutrophil homeostasis is maintained by a fine balance between granulopoiesis in the bone marrow, retention in and release from the bone marrow and clearance and destruction. This review discusses the molecular mechanisms regulating neutrophil mobilization from the bone marrow, with emphasis on the antagonistic roles of the CXCR4 (C-X-C motif receptor 4)/CXCL12 (C-X-C motif ligand 12) and CXCR2/ELR+ (Glu-Leu-Arg) CXC chemokine signaling axes in the bone marrow. A role for the
\end{abstract}

CXCL12/CXCR4 chemokine axis in the trafficking of senescent neutrophils back to the bone marrow for clearance, along with the role of bone marrow macrophages and the molecules that mediate neutrophil clearance by bone marrow macrophages, is also discussed.

Copyright $\odot 2013$ S. Karger AG, Basel

Neutrophils are an essential component of the innate immune response and the first line of defense against a wide array of invading pathogens. These cells constitute one of the main cellular components for the destruction of infectious microorganisms and, as a consequence, persistent neutropenia or hereditary deficiencies are associated with a marked susceptibility to infection. However, neutrophil accumulation and activation in tissues can have a deleterious impact, as seen in a number of inflammatory diseases, such as rheumatoid arthritis, acute respiratory stress syndrome, postischemic injury, and chronic obstructive pulmonary disease. Thus, it is very important that the level of neutrophils in the blood is regulated, both to control infections and to prevent their potential damaging effect in tissues. Neutrophil homeostasis can be regulated at several levels: neutrophil production in the bone marrow, release from the bone marrow

\section{KARGER}

E-Mail karger@karger.com

www.karger.com/jin
(C) 2013 S. Karger AG, Basel

$1662-811 \mathrm{X} / 13 / 0054-0304 \$ 38.00 / 0$
Prof. Sara M. Rankin

Leukocyte Biology Section, National Heart and Lung Institute

Faculty of Medicine, Imperial College London

London SW7 2AZ (UK)

E-Mail s.rankin@imperial.ac.uk 
to blood, distribution between the marginated pool and the freely circulating pool in the blood, as well as half-life in and clearance from the blood.

\section{Neutrophil Production}

Neutrophil production and differentiation is confined to the bone marrow and involves a process of cellular proliferation and differentiation termed 'granulopoiesis'.

Discussion of the molecular pathways regulating neutrophil differentiation goes beyond the scope of this review but has been reviewed in Sigurdsson et al. [1]. The cytokine, granulocyte colony-stimulating factor (G-CSF) has been shown to play a critical role in granulopoiesis. G-CSF induces myeloid differentiation, stimulates proliferation of granulocytic precursors and has been shown to mobilize neutrophils from the bone marrow [2]. In the bone marrow, granulopoiesis is restricted to the extravascular space where hematopoietic cells form dense cords. These hematopoietic cords are interspersed among the venous sinuses and are intimately associated with stromal cells, resident macrophages and components of the extracellular matrix [3]. After their production, terminally differentiated, mature neutrophils remain in the bone marrow for a further 4-6 days, forming a storage pool, termed 'the bone marrow reserve', from which these cells can be rapidly mobilized in response to infection or an inflammatory episode [3]. Neutrophils are the most abundantly produced leukocyte in the bone marrow. Precise studies have measured a postmitotic pool of $5.59 \pm 0.9 \times 10^{9}$ neutrophils $/ \mathrm{kg}$ bodyweight in humans, from which an estimated $0.87 \times 10^{9}$ cells $/ \mathrm{kg}$ are released each day under basal conditions [3]. Recently, it has been shown that the neutrophil serine protease inhibitor, SerpinB1, is critical for the maintenance of the neutrophil reserve in the bone marrow. Serpin $B 1^{-/-}$mice exhibit a severe bone marrow neutropenia comparable to that of $g$-csf $f^{-/}$mice. The mechanism by which SerpinB1 protects mature neutrophils is not known, but it is thought that SerpinB1 regulates a proteolytic event important for neutrophil survival [4].

\section{Neutrophil Mobilization in Response to Inflammation}

Increase in blood neutrophil numbers is a well-known clinical characteristic of inflammatory disease. In mice, the neutrophil constitutes approximately $30 \%$ of the total circulating leukocytes. While absolute numbers vary between mouse strains in BALB/cJ (6-8 weeks), there are around $1.5 \times 10^{9} / 1$ neutrophils in the circulation. This increases around 4.5 fold within a matter of hours to $9 \times$ $10^{9} / 1$ after a single administration of thioglycollate to simulate an acute inflammatory response. Intravenous administration of CXCR2 (C-X-C motif receptor 2) agonists also acts to mobilize neutrophils from the bone marrow, increasing circulating neutrophil numbers to around $14 \times 10^{9} / 1$ in a couple of hours. These increases in blood neutrophils correspond with reduced numbers of neutrophils in the bone marrow [5]. In humans, neutrophils make up around $70 \%$ of the total circulating leukocytes. The neutrophil pool has been estimated to be around $4.3 \times 10^{9} / 1$ in males and $4.5 \times 10^{9} / 1$ in females with environmental and genetic backgrounds accounting for differences between individual populations [6]. Intravenous administration of a bacterial endotoxin such as Escherichia coli increases the numbers of circulating neutrophils to around $10 \times 10^{9} / 1$ within $6 \mathrm{~h}$ [7].

\section{CXCR4/CXCL12 in the Retention, Mobilization and Clearance of Neutrophils}

Several lines of evidence suggest that CXCL12 (C-X-C motif ligand 12)/CXCR4 signaling is central to the retention of neutrophils in the bone marrow, their mobilization from the bone marrow and the homing back of senescent neutrophils to the bone marrow [8-10].

\section{CXCR4/CXCL12 Mediates Neutrophil Retention in the Bone Marrow}

The bone marrow acts as a large reservoir of mature neutrophils. It is estimated that during the homeostatic state, more than $90 \%$ of total neutrophils are held within the bone marrow, with only $1-2 \%$ in circulation $[2,3]$. This suggests that there are specific molecular mechanisms regulating the retention of these cells in the bone marrow.

CXCL12, also known as stromal cell-derived factor $1 \alpha$, was originally cloned from the culture supernatant of a bone marrow stromal cell line [5]. CXCL12 is constitutively produced by bone marrow stromal cells, including vascular endothelial cells and osteoblasts [3]. Recently, studies performed using CXCL12-green fluorescent protein knock-in mice identified a distinct population of reticular cells in the bone marrow which express high levels 
of CXCL12. These cells were termed 'CXCL12-abundant reticular cells' (CAR cells) and, under homeostatic conditions, they are the main producers of CXCL12 in bone marrow [11]. Immunohistochemical analysis of CXCL12-green fluorescent protein knock-in mice revealed that CAR cells have several long processes and that all bone marrow sinusoidal endothelial cells are surrounded by a proportion of CAR cells [11].

While CXCL12 is produced constitutively in the bone marrow, it was recently shown that the expression of CXCL12 is regulated by genes that are subject to circadian rhythms. Adrenergic signals are locally delivered by sympathetic nerves in the bone marrow, signaling via the $\beta_{3}$-adrenergic receptor on stromal cells, leading to a decreased nuclear content of the Sp1 transcription factor and the rapid downregulation of CXCL12. Thus, the expression of CXCL12 is cyclical, in rhythm with adrenergic signals delivered to stromal cells of the bone marrow by the sympathetic nervous system [12]. Interestingly, blood leukocyte numbers also exhibit circadian oscillations [13]. Circulating leukocyte numbers in murine blood peak $5 \mathrm{~h}$ after the onset of light (Zeitgeber time, ZT5) and reach a trough at ZT13 [14]. Peak recruitment of leukocytes to the site of tissue injury occurs at night in mice, during periods of activity, and is tied in with the cyclical expression of intercellular adhesion molecule 1 and CCL2 (C-C motif ligand 2) on endothelial cells at the site of inflammation [14]. Conversely, the number of endogenous rolling leukocytes in bone marrow sinusoids is increased at ZT13 compared to ZT5, and this overlaps with oscillations in the expression of P-selectin, E-selectin and vascular cell adhesion molecule 1 on bone marrow endothelial cells, promoting their homing back to the marrow [14].

CXCL12 exerts a broad spectrum of biological effects on many leukocytes and is a chemoattractant for monocytes, hematopoietic progenitor cells and subpopulations of $\mathrm{B}$ and $\mathrm{T}$ lymphocytes. It is constitutively expressed at high levels in the bone marrow and lung and at lower levels in almost all other tissues [3].

The biological actions of CXCL12 are mediated through its interaction with the G-protein-coupled, 7-transmembrane receptor CXCR4. CXCL12 has also been shown to bind with high affinity to the CXCR7 receptor, although classical G-protein-coupled signaling has not yet been demonstrated for this receptor [15]. Instead, CXCR7 appears to act as a scavenger, sequestering and internalizing excess CXCL12 and CXCL11, creating functional chemotactic gradients [16]. This function of CXCR7 was first shown to be essential for the CXCR4- mediated migration of primordial germ cells and lateral line primordium in zebrafish [16]. Studies of mice with a genetic deletion of CXCR7 have shown that this chemokine receptor is also required for cardiac semilunar valve morphogenesis and bone morphogenetic protein signaling [16]. To date, CXCR7 has not been shown to play a role in neutrophil biology.

While peripheral blood neutrophils express CXCR4 mRNA, cell surface expression of CXCR4 is barely detectable on neutrophils freshly isolated from the blood [3]. Mature neutrophils in the bone marrow express low but detectable levels of CXCR4, but at these levels, CXCR4 does not support chemotaxis [8].

Interestingly, it has been shown that CXCR4 receptor expression is downregulated when stably transduced HeLa, U-937 and CEM cells are grown in tissue culture medium containing high concentrations of CXCL12 [17]. Given that mature bone marrow neutrophils contain high intracellular levels of CXCR4 [8], it is likely that these cells express low levels of CXCR4 on their surface because of sustained downregulation of the receptor as a result of the high levels of CXCL12 in their bone marrow microenvironment. Indeed, high levels of intracellular CXCR4 have been reported in freshly isolated bone marrow neutrophils [8].

Experiments performed in mice, using the CXCR4 antagonist, AMD3100, has shown that antagonism of CXCR4 results in a 3-fold increase in the number of circulating neutrophils and that this increase corresponded to a reduction in bone marrow neutrophil numbers [8], suggesting that the increase in circulating blood neutrophils was due to their release from the bone marrow. Similar experiments were performed in healthy human volunteers, and it was found that infusion with AMD3100 stimulated a dose-related increase in the number of circulating neutrophils, that begins within an hour and peaks between 3 and $6 \mathrm{~h}$, with a maximum increase of 2.7 fold [18]. When lethally irradiated wild-type (WT) mice were repopulated with CXCR4-deficient fetal liver cells, hematopoiesis was fully reconstituted 4 weeks after transplant. However, compared to mice reconstituted with WT cells, it was observed that neutrophil numbers in the bone marrow of CXCR4-deficient mice were markedly reduced while, at the same time, the absolute number of neutrophils in the blood was increased [9].

Further, mice carrying a myeloid-specific deletion of CXCR4 [myeloid-specific knock-out (MKO) mice], in which CXCR4 in mature neutrophils is deleted in both bone marrow and blood, display a marked redistribution of neutrophils from the bone marrow to the blood and 
spleen [19]. Interestingly, there was no increase in the number of immature myeloid cells in the blood, suggesting that CXCR4 expression is important for the retention of mature, but not myeloid, progenitor neutrophils in the bone marrow, and the increase observed in the circulation corresponded to a decrease in mature neutrophils in the bone marrow. It was also shown that blood neutrophilia was not a result of increased granulopoiesis as the number of granulocytic precursors and cytokine responsiveness of myeloid progenitors was comparable in $\mathrm{MKO}$ and control mice [19].

Similar to MKO mice, mice with a conditional deletion of CXCL12 display a significant increase in the number of circulating neutrophils [20].

Evidence suggests that during homeostasis, G-CSF regulates both granulopoiesis and neutrophil mobilization from the bone marrow [2]. The mobilization from the bone marrow by G-CSF may be facilitated by the effects of G-CSF on CXCR4/CXCL12 signaling. Daily treatment with G-CSF for 4-5 days results in a progressive decrease in CXCL12 mRNA in the bone marrow [2]. This decrease in CXCL12 mRNA is closely mirrored by a decrease in CXCL12 protein expressed in the bone marrow as well as an increase in neutrophil release from the bone marrow [2]. In a mouse model of chronic G-CSF treatment $(250 \mu \mathrm{g} / \mathrm{kg}$ twice daily for 6 consecutive days), the concentration of CXCL12 in the bone marrow decreases significantly between days 2 and 6 of G-CSF treatment. This decrease corresponds to an accumulation of serine proteases able to directly cleave and inactivate CXCL12, suggesting that G-CSF treatment mobilizes neutrophils from the bone marrow by activating proteases that cleave CXCL12 [21]. However, mice lacking dipeptidyl peptidase I, an enzyme required for the functional activation of many serine proteases, retain the ability to mobilize neutrophils [22]. Interestingly, treatment with G-CSF also induces a decrease in the surface expression of CXCR4 on neutrophils over 5-18 h [23]. More recent studies, by others, investigating the mechanisms whereby G-CSF treatment over 4 days mobilizes hematopoietic stem cells have similarly shown a role for reduced expression of CXCL12 and found that bone marrow macrophages are critical in regulating CXCL12 production by stromal cells $[24,25]$.

Inflammation in animal models and humans is associated with an increase in serum levels of G-CSF, and intravenous injection of G-CSF induces a rapid increase in blood neutrophil numbers within a matter of hours [5]. Furthermore, G-CSF blockade in a model of acute peritonitis abrogated neutrophil mobilization from the bone marrow [5]. Interestingly, an intraperitoneal injection of G-CSF stimulated a rapid increase in circulating neutrophil numbers but did not stimulate neutrophil recruitment into the peritoneum, suggesting that GCSF production at the site of inflammation acts systemically to stimulate neutrophil mobilization from the bone marrow to the circulation but not recruitment to inflamed tissues [5]. Recently, it has been shown that this acute mobilization of neutrophils from bone marrow in response to G-CSF is dependent on CXCR2, suggesting another indirect mechanism whereby G-CSF regulates neutrophil mobilization from the bone marrow [26].

Warts, hypogammaglobulinemia, infections and myelokathexis (WHIM) syndrome is a rare congenital immune deficiency disorder, characterized by peripheral neutropenia, despite normal and often increased (myelokathexis) numbers of mature neutrophils in the bone marrow. These mutations result in desensitization and inefficient internalization of CXCR4 and consequent hyperresponsiveness to CXCL12 [27]. WHIM is most commonly caused by gain of function mutations that truncate the $\mathrm{C}$ terminus of CXCR 4 by $10-19$ amino acids, a mutation in CXCR4 ${ }^{\mathrm{R} 334 \mathrm{X}}$, although, most recently, another mutation, CXCR4 $4^{\mathrm{E} 343 \mathrm{~K}}(1027 \mathrm{G} \rightarrow \mathrm{A})$, was also identified [28]. Therefore, neutropenia seen in patients with WHIM syndrome may be a result of chronic CXCL12/CXCR4 signaling in neutrophils and their abnormal retention in bone marrow. Peripheral neutrophil numbers in these patients can be restored to normal levels by administration of G-CSF [3], and recently, it was shown that the CXCR4 antagonist, AMD3100, could also effectively treat the panleukopenia seen in patients with WHIM syndrome [29]. A study investigating neutrophil trafficking in a zebrafish model of WHIM syndrome showed abnormal CXCL12-dependent neutrophil retention in hematopoietic tissue [30]. In this case, depletion of CXCL12 restored neutrophil chemotaxis to inflammatory sites.

\section{CXCR2 Ligands Act to Mobilize Neutrophils from the Bone Marrow}

To exit the bone marrow neutrophils must migrate through the sinus wall composed of endothelial cells, which form the blood/bone marrow barrier, a discontinuous basement membrane and a continuous layer of adventitial cells. Migration across the bone marrow endothelium occurs through tight-fitting pores by a process of 
transcellular migration. In this process, neutrophils migrate through the cell body of the endothelium, rather than at cell-cell junctions [31].

The discovery that CXCL1 (KC) acts synergistically with AMD3100 to mobilize neutrophils suggests that there are other mechanisms that regulate neutrophil mobilization from the bone marrow [8]. The chemokines CXCL1 and CXCL2 (MIP-2/GRO $\beta$ ) are potent chemoattractants for neutrophils, and increased serum levels of these chemokines are a hallmark of infection and inflammation in peripheral tissues. CXCL1 and CXCL2 play an important role in the recruitment of neutrophils from the circulation to the site of inflammation [32]. An injection of CXCL1 or CXCL2, either intravenously or peripherally, to simulate a model of acute inflammation, causes selective and rapid mobilization of neutrophils from the bone marrow [5, 31]. RNA expression profiling of bone marrow cells shows that CXCL1 and CXCL2 are constitutively expressed in osteoblasts and endothelial cells, with higher expression in endothelial cells, and that treatment with G-CSF causes an approximately 2.7 -fold increase in CXCL1 production by endothelial cells [26]. It was recently discovered that megakaryocytes positioned next to sinus vessels in the bone marrow also produce CXCL1 and that G-CSF indirectly triggers the release of CXCL1 from these megakaryocytes [33]. In this study, the authors report a peak in the serum levels of CXCL1, $2 \mathrm{~h}$ after a single intravenous injection of G-CSF, and suggest that G-CSF acts to increase thrombopoietin levels in bone marrow that in turn stimulates the release of CXCL1 from megakaryocytes [33].

CXCR2, the sole receptor for CXCL1 and CXCL2 in mice, is expressed on mature neutrophils in the bone marrow and blood and is important for the mobilization of neutrophils from the bone marrow. The study by Köhler et al. [33] highlights the essential role of CXCR2 in neutrophil mobilization from the bone marrow, as GCSF injections were unable to mobilize neutrophils from the bone marrow of CXCR2-deficient mice. Treatment of naïve mice with a small molecule CXCR2 antagonist, for $2 \mathrm{~h}$, decreases neutrophil numbers in the blood and inhibits neutrophil mobilization in response to an intravenous injection of KC (N. Strydom, personal communication). Other studies carried out with $\mathrm{CxCr} 2^{-/-}$mice have given conflicting results, as $\mathrm{Cxcr} 2^{-/-}$mice housed under specific pathogen-free conditions display blood neutrophilia, whereas $\mathrm{CxCr} 2^{-/-}$mice housed under gnotobiotic conditions have normal numbers of circulating neutrophils [32]. To overcome this issue, Eash et al. [26] generated a mixed bone marrow chimera reconstituted with
$\mathrm{Cxcr}^{-/-}$and WT hematopoietic cells to study the cellintrinsic role of CXCR2 in neutrophil mobilization from the bone marrow. They found that the number of $\mathrm{CxCr} 2^{-/-}$ neutrophils in the blood of these mice was significantly reduced compared with WT mice, suggesting that ELR+ (Glu-Leu-Arg) CXC chemokines may be required for neutrophil mobilization under homeostatic as well as inflammatory conditions. Eash et al. [26] found that shortterm G-CSF administration failed to mobilize $\mathrm{Cxcr} 2^{-/-}$ neutrophils into the blood, showing that maximal neutrophil responses to G-CSF requires CXCR2. These studies provide compelling evidence that CXCR2 signaling has an important role in the mobilization of neutrophils from the bone marrow under inflammatory and homeostatic conditions.

\section{CXCR2 and CXCR4 Signaling Antagonistically Regulate Neutrophil Mobilization}

The first evidence for interaction between CXCR2 and CXCR4 signaling came from a study that reported that coincubation of human neutrophils with CXCL12 reduced the shape change seen when neutrophils are stimulated with CXCR2 ligands [8]. In naïve mice, coadministration of $\mathrm{KC}$ and the CXCR4 antagonist, AMD3100, stimulated an additive effect with respect to mobilization [8]. Furthermore, the chemotaxis of murine bone marrow-derived neutrophils toward $\mathrm{KC}$ was significantly inhibited when the neutrophils were incubated in the presence of CXCL12 during the assay [8]. In irradiated chimeric mice reconstituted with WT and $C x c r 2^{-/-}$myeloid cells, AMD3100 was unable to mobilize $\mathrm{Cxcr} 2^{-/-}$neutrophils from the bone marrow of these mice, suggesting that the neutrophil mobilization seen in response to transient CXCR4 blocking is dependent on CXCR2 expression on bone marrow neutrophils [26]. In mice reconstituted with WT and Cxcr4-/- myeloid cells (MKO mice), an intravenous injection of the CXCR2 agonist, MIP-2, caused only a minimal increase in the circulating number of $\mathrm{CxCr}^{-/-}$neutrophils whereas MIP-2 induced an approximately 4 -fold increase in the number of WT neutrophils in these mice [26].

The data summarized here suggest an interplay between CXCR2 and CXCR4 in regulating neutrophil mobilization. CXCR2 ligand-producing endothelial cells and megakaryocytes direct neutrophil chemotaxis to the vasculature for entry into the circulation, while CXCL12producing CAR cells and osteoblasts promote their retention in the bone marrow (fig. 1). 
Fig. 1. Neutrophil mobilization from the bone marrow during homeostasis and inflammation. During homeostasis, the high concentrations of CXCL12 produced by CAR cells and osteoblasts in the bone marrow act to retain neutrophils in the bone marrow via CXCR4 expressed on neutrophils, whilst low concentrations of ELR+ CXC chemokines expressed on endothelial cells and produced by megakaryocytes allow steady-state mobilization of neutrophils via CXCR2. During inflammation, the concentration of CXCR2 ligands increases in the blood while CXCL12 expression in the bone marrow decreases, allowing increased neutrophil migration towards ELR+ CXC chemokines.

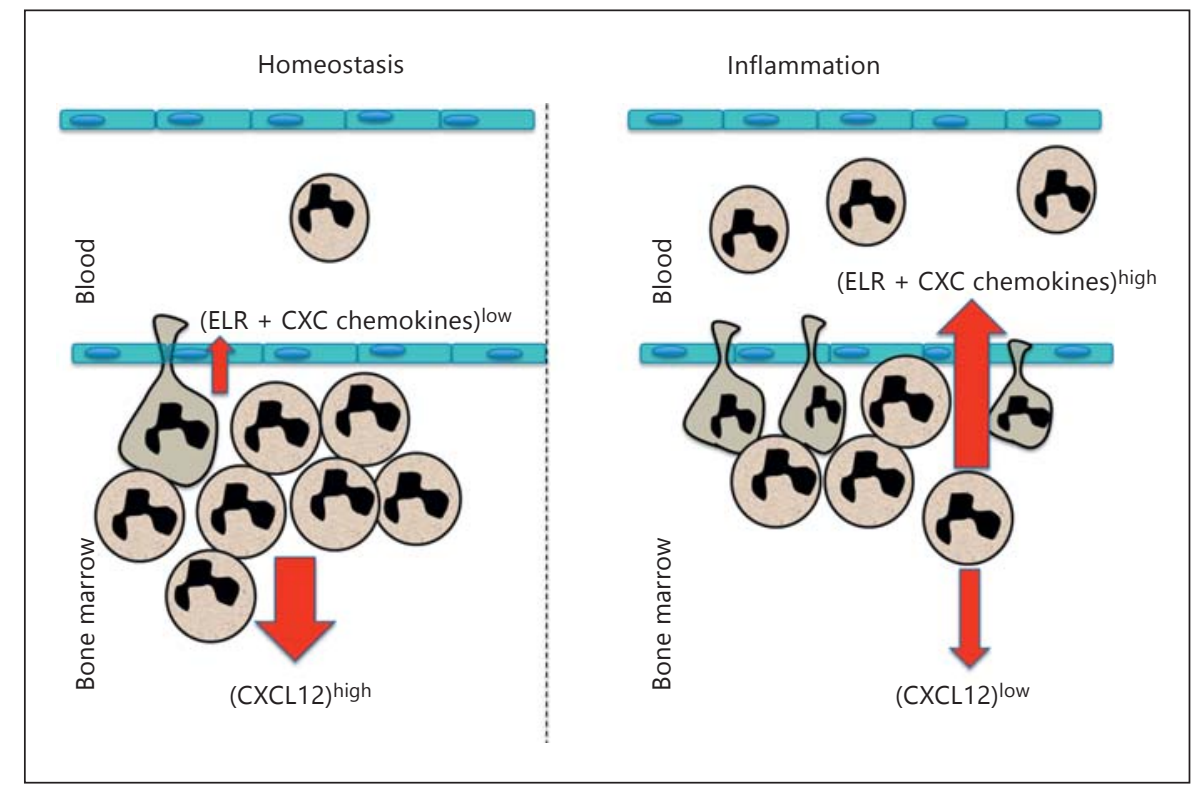

\section{Modulation of Surface CXCR4 Expression on Neutrophils as They Age}

When human, peripheral blood neutrophils are cultured for $20 \mathrm{~h}$, their surface expression of CXCR4 is dramatically upregulated [10]. Similar observations were made when murine bone marrow neutrophils were aged in vitro for 6 and $20 \mathrm{~h} \mathrm{[8].} \mathrm{CXCR4} \mathrm{expression} \mathrm{was} \mathrm{seen} \mathrm{to}$ increase dramatically after $6 \mathrm{~h}$ and reached a maximum after $20 \mathrm{~h}$, at which point around $76 \%$ of the neutrophil population had become apoptotic. These CXCR $4^{\text {high }}$ neutrophils have since been termed 'senescent' and may represent a distinct neutrophil phenotype, as CXCR4 $4^{\text {high }}$ neutrophils also show enhanced migratory responses to CXCL12 as well as reduced expression of CXCR2 and migration to CXCL1. These changes in chemokine receptor expression precede the upregulation of markers of apoptosis such as CD16 and annexin V, making this CXCR4 ${ }^{\text {high }}$ population distinct from both freshly isolated and apoptotic neutrophils. Moreover, in contrast to senescent neutrophils, apoptotic neutrophils lose their ability to migrate to CXCL12 despite maintaining high levels of surface CXCR4.

As CAR cells in the bone marrow produce high levels of CXCL12, it is feasible that senescent neutrophils that express CXCR4 could home back to the bone marrow. Indeed, it was found that CXCR $4^{\text {high }}$ neutrophils preferentially homed to the bone marrow. Confocal microscopy revealed that these cells homed to the hematopoietic compartment of the bone marrow, suggesting that they were able to attach to and migrate through the sinusoidal endothelium and into the stromal compartment [8]. When CXCR $4^{\text {low }}$ and CXCR $4^{\text {high }}$ neutrophils were infused into naïve mice, approximately 5.6 times more CXCR4 ${ }^{\text {high }}$ neutrophils were sequestered in the bone marrow than CXCR4 $4^{\text {low }}$ neutrophils. This sequestration of senescent neutrophils in the bone marrow was significantly reduced, both by direct blocking and by desensitization of the CXCR4 receptors, indicating that homing is mediated via CXCR4 [8]. Bone marrow neutrophils isolated from MKO mice are made up of a mixed population of CXCR $4^{+}$ and CXCR4 ${ }^{-}$neutrophils. When this mixed population is adoptively transferred into naïve mice, they show reduced homing to the bone marrow, confirming that CXCR4 contributes to neutrophil homing to the bone marrow [19].

\section{Clearance of Senescent Neutrophils in the Bone Marrow}

Several studies have reported a circulating neutrophil half-life of about $8 \mathrm{~h}$ in humans and $13.7 \mathrm{~h}$ in mice. However, a study using in vivo labeling found that under homeostatic conditions, neutrophils may circulate in the blood for an average of 5.4 days in humans and $90 \mathrm{~h}$ in mice [7]. These results differ from a significant number of previous studies which have shown much shorter life- 
spans in the circulation, and some authors have cast doubts on the validity of the mathematical modeling used by Pillay et al. [7], specifically suggesting that the results do not account for the long transit time of neutrophils in the bone marrow [34].

When we consider that $0.89 \times 10^{9}$ cells $/ \mathrm{kg}$ bodyweight of these cytotoxic neutrophils are released into the blood per day, the need for an efficient removal system is evident [19]. Neutrophils die by a process of apoptosis, and the expression of certain surface molecules such as phosphatidyl serine (PS), by apoptotic neutrophils lead to their recognition and phagocytosis by macrophages either present at inflammatory sites or, during the homeostatic state, by macrophages present at sites of neutrophil clearance [35]. It is traditionally considered that the liver and spleen are the primary sites of neutrophil clearance [3]. However, studies in mice and man have provided evidence that in addition to the spleen and liver, the bone marrow may also contribute substantially to the clearance of neutrophils from the circulation $[3,36]$.

Over the past 50 years, experiments studying neutrophil kinetics in vivo have often made use of radiolabels [37-39]. Findings from these early experiments form the foundations of our knowledge of neutrophil kinetics, and, if we look closely, they also provide evidence that a large proportion of neutrophils home from the circulation back to the bone marrow. In one such study, peripheral blood neutrophils were isolated from 15 patients and labeled with Indium-111 ( $\left.{ }^{111} \mathrm{In}\right)$. The labeled neutrophils were then administered to the patients and the radioactivity measured for up to $48 \mathrm{~h}$ after injection by means of a largefield gamma camera. When acquiring the data, the authors focused on the liver and spleen, but also took in a part of the lower spine, where they were able to detect some radioactivity [39]. A more comprehensive study in mice examined the localization of infused ${ }^{111} \mathrm{In}$-labeled murine peripheral blood neutrophils, peritoneal inflammatory exudate neutrophils and mature bone marrow neutrophils in vivo [36]. After $4 \mathrm{~h}$, peripheral blood neutrophils homed significantly to both the bone marrow (approximately $31 \%$ ) and liver (approximately $32 \%$ ). Bone marrow neutrophils predominantly homed to the bone marrow (approximately 66\%) and liver (approximately $15 \%)$, whereas inflammatory exudate neutrophils homed primarily to the liver (approximately $42 \%$ ), with modest localization in the bone marrow (20\%). Sequestration in the spleen was similar for all three neutrophil populations. Further investigation revealed that the labeled cells in the bone marrow were closely associated with cells of myelomonocytic lineages [36]. Interestingly, the authors also found that marrow-sequestered labeled neutrophils of peripheral blood origin could not be remobilized from the bone marrow following sterile peritonitis induction, indicating that movement of the more mature peripheral blood neutrophils is unidirectional and suggesting that the bone marrow is the final destination of those cells [36]. Another study determined the relative contribution of the bone marrow to the clearance of senescent neutrophils by aging isolated bone marrow neutrophils in vitro, radiolabeling with ${ }^{111}$ In and injecting intravenously into recipient mice [40]. Using these methods, it was determined that after $2 \mathrm{~h}$, approximately $34 \%$ radioactivity was contained within the bone marrow, approximately $29 \%$ in the spleen and approximately $27 \%$ in the liver [40]. Consistent with the role of the CXCR4/CXCL12 chemokine axis in the homing of neutrophils to the bone marrow, it was also found that pretreatment with pertussis toxin, a bacterial toxin that specifically inhibits $\mathrm{G}$ alpha $\mathrm{i}_{\mathrm{i}}$, dramatically inhibited neutrophil accumulation in the bone marrow [40].

\section{Bone Marrow Macrophages Phagocytose Apoptotic Neutrophils}

If neutrophils were to die by necrosis and disintegrate in situ, the release of their cytotoxic contents may trigger an inflammatory response and cause extensive tissue damage. However, it is known that both in the inflammatory and homeostatic states, neutrophils become apoptotic and are phagocytosed by macrophages [35]. In addition, the phagocytosis of apoptotic neutrophils by macrophages at sites of inflammation reduces their production of proinflammatory chemokines and stimulates the release of potent anti-inflammatory mediators such as prostaglandin $\mathrm{E}_{2}$ and transforming growth factor- $\beta_{1}$ [35].

As mentioned, Suratt et al. [36] found that radiolabeled neutrophils sequestered in the bone marrow were closely associated with cells of myelomonocytic lineages. In the liver and spleen, macrophages of the endoreticular system directly recognize and phagocytose circulating apoptotic neutrophils. In the bone marrow, macrophages are situated in the hematopoietic cords, separated from the blood by the bone marrow sinusoidal endothelium. Bone marrow macrophages provide cell-cell contact necessary for lymphopoiesis and hematopoiesis [3]. They are also important phagocytic cells, responsible for clearing nuclei expelled by developing erythroblasts during erythropoiesis and pre- $\mathrm{B}$ cells with nonproductive $\mathrm{B}$-cell receptor rearrangements [3]. It is known that during embryogenesis and before the establishment of hematopoi- 


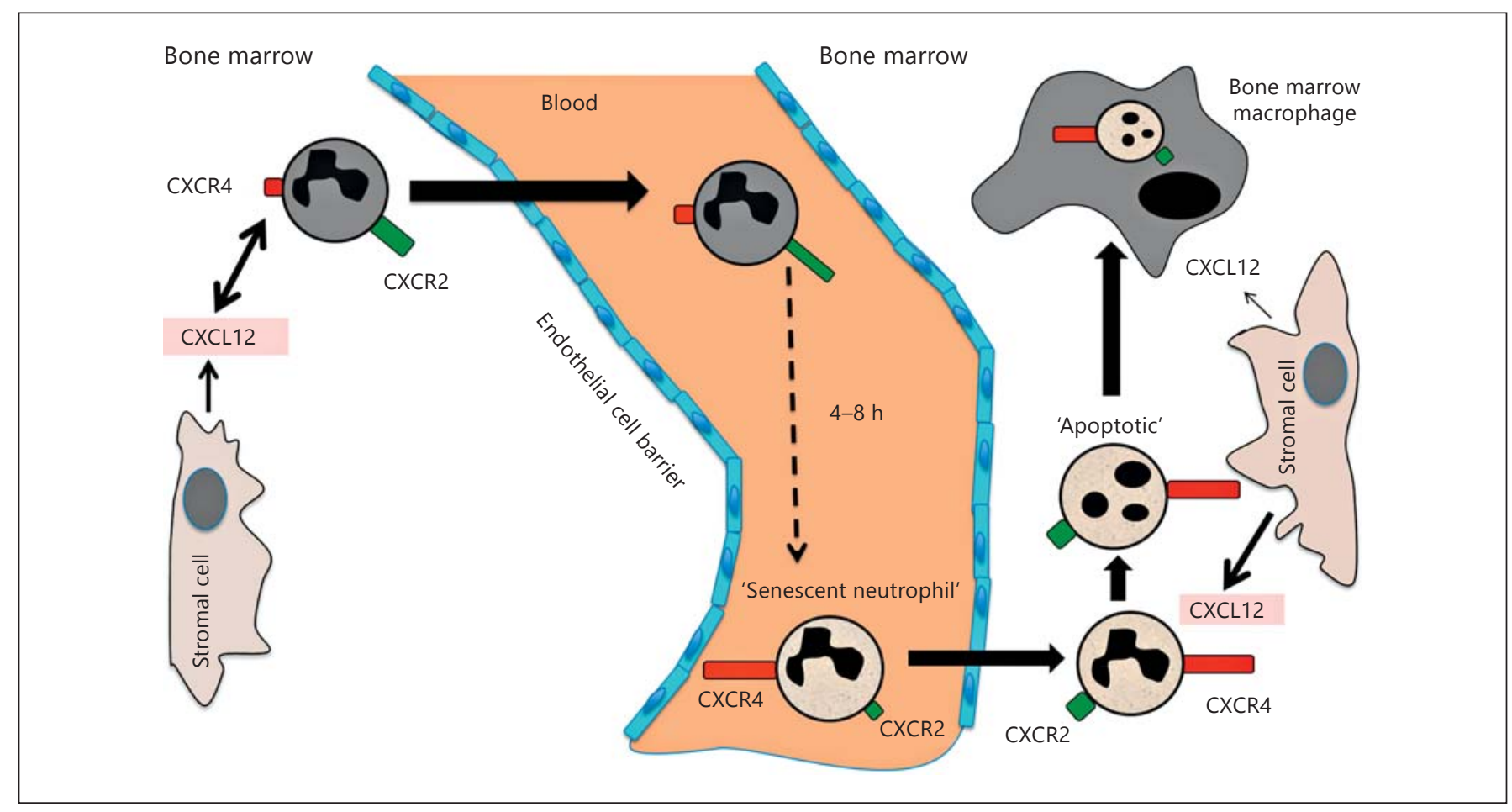

Fig. 2. Neutrophil clearance in the bone marrow. Within hours of their release into the blood, neutrophils start to upregulate their expression of CXCR4. The chemotactic gradient that results as a consequence of bone marrow stromal cells constitutively produc- ing CXCL12 now allows CXCR4 ${ }^{\text {high }}$ senescent neutrophils to home back to the bone marrow where they become apoptotic and are phagocytosed by bone marrow macrophages. esis, the bone marrow serves as the site of neutrophil destruction [41]. Electron microscopy of adult human bone marrow also reveals the presence of apoptotic neutrophils within bone marrow macrophages [42].

In a mouse study, it was shown that $24 \mathrm{~h}$ after injection, adoptively transferred neutrophils labeled with $0.5-\mu \mathrm{m}$ inert latex microspheres were phagocytosed by bone marrow macrophages, as macrophages were found to contain both latex microspheres and apoptotic bodies [40]. Moreover, $2 \mathrm{~h}$ after adoptive transfer, most latex-labeled cells in the bone marrow are neutrophils, whereas $24 \mathrm{~h}$ after adoptive transfer, $50 \%$ of labeled cells are macrophages [40]. These data provide direct evidence that senescent neutrophils are recruited to the bone marrow where they undergo apoptosis and are subsequently phagocytosed by stromal macrophages (fig. 2). These results are further supported by a recent study showing that neutrophil clearance is delayed in genetically modified mice lacking the antiapoptotic gene cellular FLICE-like inhibitory protein and exhibit a selective reduction in macrophages in the bone marrow and marginal zone of the spleen [43]. A recent human study looking at the whole-body redistri- bution of ${ }^{111}$ In-labeled neutrophils $45 \mathrm{~min}, 24 \mathrm{~h}$ and 2, 4, 7 and 10 days after administration, provides further evidence that neutrophils, having homed to the bone marrow, do not reenter the circulation, as no further distribution of administered neutrophils could be detected $24 \mathrm{~h}$ after administration [44]. Interestingly, in this study, patients with chronic obstructive pulmonary disease demonstrated increased clearance of neutrophils in their bone marrow ( $80 \%$ compared with $67 \%$ in healthy subjects). The authors suggested that this may be due to altered bone marrow physiology resulting from bone marrow stimulation or chronic hypoxemia. Patients with asthma and bronchiectasis showed levels of bone marrow neutrophil clearance comparable with healthy nonsmokers [44].

The uptake of apoptotic neutrophils by bone marrow macrophages was shown to stimulate their production of G-CSF [40]. As G-CSF drives basal neutrophil granulopoiesis in the bone marrow, it is possible that the homing of aged neutrophils to the bone marrow represents a positive feedback loop that regulates the rate of granulopoiesis during homeostatic conditions, though this needs to be proven experimentally. 
Another body of work has suggested that interleukin (IL)-17 and IL-23 regulate granulopoiesis $[45,46]$. Stark et al. [45] showed that phagocytosis of apoptotic neutrophils suppresses IL-23 secretion from phagocytes, which in turn reduces IL-17 production by IL-23 receptor-bearing $\mathrm{T}$ cells. The resulting decrease in serum levels of IL-17 reduces the production of G-CSF in the bone marrow, thereby inhibiting neutrophil production and mobilization [45]. The neutrophilia observed in $\mathrm{CXCR}^{-1-}$ mice has similarly been associated with IL17 , as these mice exhibit abnormally high levels of plasma IL-17 [47]. In another study, the authors reported an accumulation of apoptotic neutrophils in the spleen and liver of liver $\mathrm{X}$ receptor (LXR)-deficient mice $\left(\mathrm{LXR} \alpha / \beta^{-/-}\right)$[46]. Activation of LXR in cultured WT bone marrow-derived dendritic cells strongly reduced IL-23 expression by these cells, and engulfment of apoptotic neutrophils significantly decreased lipopolysaccharide-induced IL-23 expression in WT macrophages but not in LXR $\alpha / \beta^{-/}$macrophages, suggesting that LXR signaling downstream of apoptotic cell engulfment is needed for the repression of IL-23 expression [46]. Interestingly, LXR signaling does not influence neutrophil clearance in the bone marrow [46]. It is important to note that the studies that have implicated the IL-23/IL-17 axis in the regulation of granulopoiesis have utilized thioglycollate-elicited peritoneal macrophages and, in the case of the paper by Stark et al. [45], adhesion molecule-deficient mice exhibited high basal neutrophilia associated with high serum levels of IL-17 and G-CSF, complicating interpretation of these results, with respect to homeostasis. Moreover, in naïve mice, blockade of G-CSF, but not IL-17, reduces neutrophil numbers in the blood and, in IL-17 receptor-deficient mice, no effects on basal circulating neutrophil numbers have been reported $[40,48]$.

\section{Molecules That Mediate the Recognition and Engulfment of Neutrophils by Bone Marrow Macrophages}

Apoptosis is associated with cell surface changes that promote recognition and phagocytosis by macrophages. Neutrophil apoptosis is accompanied by the increased expression of PS and the reduced expression of adhesion molecules and receptors, including CXCR2, involved in triggering effector functions [49].

PS is relocated from the inner to the outer leaflet of the plasma membrane during apoptosis, and original studies showed that PS is necessary for the recognition of both murine and human apoptotic neutrophils by thioglycollate-elicited peritoneal murine macrophages $[49,50]$. However, it is known that different populations of macrophages use different receptors to recognize and remove apoptotic cells. The process of apoptotic cell clearance by macrophages is considered critical for the resolution of an inflammatory response and, whereas a discussion of the molecules and mechanisms involved is beyond the scope of this review, the molecules that mediate the uptake of apoptotic cells by macrophages is discussed in detail in Bratton and Henson [51]. Whereas PS and PS derivatives can inhibit the phagocytosis of apoptotic cells by inflammatory macrophages, they have no effect on the recognition and phagocytosis of apoptotic neutrophils by murine bone marrow macrophages or human monocytederived macrophages [50].

An early study reported the presence of the adhesive protein, thrombospondin (TSP), in the interaction between human monocyte-derived macrophages and senescent neutrophils, associated with the macrophage surface and in solution [52]. Inhibition of the TSP receptors on macrophages, CD36 and the vitronectin receptor $\left(\alpha_{5} \beta_{3}\right)$ using monoclonal antibodies had synergistic inhibitory effects on both macrophage recognition of senescent neutrophils and macrophage adhesion to TSP [52]. Murine bone marrow macrophages, like human monocyte-derived macrophages, also use the vitronectin receptor for the removal of apoptotic cells regardless of species or type [52]. Furthermore, it has been shown that late apoptotic human neutrophils bind TSP and that TSP forms a 'molecular bridge' between the apoptotic neutrophil and the surface of the phagocyte; however, the TSPbinding moiety on apoptotic neutrophils remains to be defined [53].

A number of studies have implicated annexin A1 as a potential prophagocytic molecule $[53,54]$. More specifically, in vitro, bone marrow macrophages from annexin A1 KO (AnxA1 $1^{-/-}$) mice show $40 \%$ decreased phagocytosis of apoptotic neutrophils compared with WT controls [54]. A recent study has reported that AnxA1 ${ }^{-/-}$mice have increased numbers of neutrophils in their bone marrow and that this was not due to an increased rate of neutrophil production, but the accumulation of senescent CXCR $4^{\text {high }}$ neutrophils in the bone marrow [55]. AnxA1 ${ }^{-/-}$mice also showed significantly reduced accumulation of WT neutrophils in bone marrow macrophages in vivo when these WT neutrophils were labeled with latex microspheres and adoptively transferred into AnxA1 ${ }^{-/-}$mice, indicating an im- 
paired ability of AnxA1 $1^{-/-}$macrophages to phagocytose apoptotic neutrophils. Therefore, the increased numbers of senescent neutrophils seen in the bone marrow of $\mathrm{AnxA1}^{-/-}$mice may be due to the accumulation of senescent neutrophils homing to the bone marrow that are not efficiently cleared by the AnxA1 $1^{-/-}$bone marrow macrophages [55].

\section{Summary and Future Directions}

In recent years, unraveling the role of chemokines has enhanced our understanding of neutrophil trafficking under homeostasis and inflammation. In particular, studies have shown the importance of the interplay between
CXCL12 and ELR+ CXC chemokines generated in the bone marrow in regulating neutrophil retention, mobilization and clearance by this tissue.

However, there are still many outstanding questions. With respect to neutrophil production and release under homeostatic conditions, it is not known how G-CSF production is regulated within the bone marrow at a cellular or molecular level. Once in the circulation, neutrophil half-life is an area of controversy that still needs to be resolved. Finally, the biological significance of neutrophil clearance in specific tissues is not clear. Thus, why are a large proportion of neutrophils cleared in the bone marrow when they could all be efficiently cleared in the liver and spleen? A more precise understanding of neutrophil clearance in each of these tissues would be beneficial.

\section{References}

$>_{1}$ Sigurdsson F, Khanna Gupta A, Lawson N, 10 Nagase H, Miyamasu M, Yamaguchi M, ImanBerliner N: Control of late neutrophil-specific gene expression: insights into regulation of myeloid differentiation. Semin Hematol 1997;34:303-310.

-2 Semerad CL, Liu F, Gregory AD, Stumpf K, Link DC: G-CSF is an essential regulator of neutrophil trafficking from the bone marrow to the blood. Immunity 2002;17:413-423.

$>3$ Rankin SM: The bone marrow: a site of neutrophil clearance. J Leukoc Biol 2010;88:241251.

-4 Benarafa C, LeCuyer TE, Baumann M, Stolley JM, Cremona TP, Remold-O’Donnell E: SerpinB1 protects the mature neutrophil reserve in the bone marrow. J Leukoc Biol 2011;90: 21-29.

5 Wengner AM, Pitchford SC, Furze RC, Rankin SM: The coordinated action of G-CSF and ELR + CXC chemokines in neutrophil mobilization during acute inflammation. Blood 2008;111:42-49.

6 von Vietinghoff S, Ley K: Homeostatic regulation of blood neutrophil counts. J Immunol 2008;181:5183-5188.

7 Pillay J, Ramakers BP, Kamp VM, Loi AL, Lam SW, Hietbrink F, Leenen LP, Tool AT, Pickkers P, Koenderman L: Functional heterogeneity and differential priming of circulating neutrophils in human experimental endotoxemia. J Leukoc Biol 2010;88:211-220.

-8 Martin C, Burdon PC, Bridger G, GutierrezRamos JC, Williams TJ, Rankin SM: Chemokines acting via CXCR2 and CXCR4 control the release of neutrophils from the bone marrow and their return following senescence. Immunity 2003;19:583-593.

9 Ma Q, Jones D, Springer TA: The chemokine receptor CXCR4 is required for the retention of $\mathrm{B}$ lineage and granulocytic precursors within the bone marrow microenvironment. Immunity 1999;10:463-471. ishi M, Tsuno NH, Matsushima K, Yamamoto K, Morita Y, Hirai K: Cytokine-mediated regulation of CXCR4 expression in human neutrophils. J Leukoc Biol 2002;71:711-717.

-11 Sugiyama T, Kohara H, Noda M, Nagasawa T: Maintenance of the hematopoietic stem cell pool by CXCL12-CXCR4 chemokine signaling in bone marrow stromal cell niches. Immunity 2006;25:977-988.

12 Mendez-Ferrer S, Lucas D, Battista M, Frenette PS: Haematopoietic stem cell release is regulated by circadian oscillations. Nature 2008;452:442-447.

13 Haus E, Smolensky MH: Biologic rhythms in the immune system. Chronobiol Int 1999;16: 581-622.

14 Scheiermann C, Kunisaki Y, Lucas D, Chow A, Jang JE, Zhang D, Hashimoto D, Merad M, Frenette PS: Adrenergic nerves govern circadian leukocyte recruitment to tissues. Immunity 2012;37:290-301.

15 Thelen M, Thelen S: CXCR7, CXCR4 and CXCL12: an eccentric trio? J Neuroimmunol 2008;198:9-13.

16 Yu S, Crawford D, Tsuchihashi T, Behrens TW, Srivastava D: The chemokine receptor CXCR7 functions to regulate cardiac valve remodeling. Dev Dyn 2011;240:384-393.

17 Tarasova NI, Stauber RH, Michejda CJ: Spontaneous and ligand-induced trafficking of CXC-chemokine receptor 4. J Biol Chem 1998;273:15883-15886.

-18 Hendrix CW, Collier AC, Lederman MM, Schols D, Pollard RB, Brown S, Jackson JB, Coombs RW, Glesby MJ, Flexner CW, Bridger GJ, Badel K, MacFarland RT, Henson GW, Calandra G: Safety, pharmacokinetics, and antiviral activity of AMD3100, a selective CXCR4 receptor inhibitor, in HIV-1 infection. J Acquir Immune Defic Syndr 2004;37: 1253-1262.
19 Eash KJ, Means JM, White DW, Link DC: CXCR4 is a key regulator of neutrophil release from the bone marrow under basal and stress granulopoiesis conditions. Blood 2009;113: 4711-4719.

20 Tzeng YS, Li H, Kang YL, Chen WC, Cheng WC, Lai DM: Loss of CXCL12/SDF-1 in adult mice decreases the quiescent state of hematopoietic stem/progenitor cells and alters the pattern of hematopoietic regeneration after myelosuppression. Blood 2011; 117:429-439.

21 Levesque J-P, Hendy J, Takamatsu Y, Simmons PJ, Bendall LJ: Disruption of the CXCR4/CXCL12 chemotactic interaction during hematopoietic stem cell mobilization induced by GCSF or cyclophosphamide. J Clin Invest 2003;111:187-196.

22 Levesque JP, Liu F, Simmons PJ, Betsuyaku T, Senior RM, Pham C, Link DC: Characterization of hematopoietic progenitor mobilization in protease-deficient mice. Blood 2004; 104:65-72.

23 Kim HK, De La Luz Sierra M, Williams CK, Gulino AV, Tosato G: G-CSF down-regulation of CXCR4 expression identified as a mechanism for mobilization of myeloid cells. Blood 2006; 108:812-820.

24 Christopher MJ, Rao M, Liu F, Woloszynek JR, Link DC: Expression of the G-CSF receptor in monocytic cells is sufficient to mediate hematopoietic progenitor mobilization by G-CSF in mice. J Exp Med 2011;208: 251-260.

25 Chow A, Lucas D, Hidalgo A, Mendez-Ferrer S, Hashimoto D, Scheiermann C, Battista M, Leboeuf M, Prophete C, van Rooijen N, Tanaka M, Merad M, Frenette PS: Bone marrow CD169+ macrophages promote the retention of hematopoietic stem and progenitor cells in the mesenchymal stem cell niche. J Exp Med 2011;208:261-271.
Mechanisms Regulating Neutrophil

Mobilization and Clearance 
-26 Eash KJ, Greenbaum AM, Gopalan PK, Link DC: CXCR2 and CXCR4 antagonistically regulate neutrophil trafficking from murine bone marrow. J Clin Invest 2010;120:2423-2431.

$\checkmark 27$ Hernandez PA, Gorlin RJ, Lukens JN, Taniuchi S, Bohinjec J, Francois F, Klotman ME, Diaz GA: Mutations in the chemokine receptor gene CXCR4 are associated with WHIM syndrome, a combined immunodeficiency disease. Nat Genet 2003;34:70-74.

28 Liu Q, Chen H, Ojode T, Gao X, AnayaO’Brien S, Turner NA, Ulrick J, DeCastro R, Kelly C, Cardones AR, Gold SH, Hwang EI, Wechsler DS, Malech HL, Murphy PM, McDermott DH: WHIM syndrome caused by a single amino acid substitution in the carboxytail of chemokine receptor CXCR4. Blood 2012;120:181-189.

-29 McDermott DH, Liu Q, Ulrick J, Kwatemaa N, Anaya-O’Brien S, Penzak SR, Filho JO, Priel DA, Kelly C, Garofalo M, Littel P, Marquesen MM, Hilligoss D, Decastro R, Fleisher TA, Kuhns DB, Malech HL, Murphy PM: The CXCR4 antagonist plerixafor corrects panleukopenia in patients with WHIM syndrome. Blood 2011;118:4957-4962.

- 30 Walters KB, Green JM, Surfus JC, Yoo SK, Huttenlocher A: Live imaging of neutrophil motility in a zebrafish model of WHIM syndrome. Blood 2010;116:2803-2811.

- 31 Burdon PC, Martin C, Rankin SM: Migration across the sinusoidal endothelium regulates neutrophil mobilization in response to ELR + CXC chemokines. Br J Haematol 2008; 142: 100-108.

-32 Day RB, Link DC: Regulation of neutrophil trafficking from the bone marrow. Cell Mol Life Sci 2012;69:1415-1423.

- 33 Köhler A, De Filippo K, Hasenberg M, van den Brandt C, Nye E, Hosking MP, Lane TE, Mann L, Ransohoff RM, Hauser AE, Winter O, Schraven B, Geiger H, Hogg N, Gunzer M: G-CSF-mediated thrombopoietin release triggers neutrophil motility and mobilization from bone marrow via induction of CXCR2 ligands. Blood 2011;117:4349-4357.

- 34 Tofts PS, Chevassut T, Cutajar M, Dowell NG, Peters AM: Doubts concerning the recently reported human neutrophil lifespan of 5.4 days. Blood 2011;117:6050-6052, author reply 6053-6054.
35 Serhan CN, Savill J: Resolution of inflammation: the beginning programs the end. Nat Immunol 2005;6:1191-1197.

-36 Suratt BT, Young SK, Lieber J, Nick JA, Henson PM, Worthen GS: Neutrophil maturation and activation determine anatomic site of clearance from circulation. Am J Physiol Lung Cell Mol Physiol 2001;281:L913-L921.

37 Athens JW: Blood: leukocytes. Annu Rev Physiol 1963;25:195-212.

38 Cartwright GE, Athens JW, Wintrobe MM: The kinetics of granulopoiesis in normal man. Blood 1964;24:780-803.

39 Thakur ML, Lavender JP, Arnot RN, Silvester DJ, Segal AW: Indium-111-labeled autologous leukocytes in man. J Nucl Med 1977;18: 1014-1021.

40 Furze RC, Rankin SM: The role of the bone marrow in neutrophil clearance under homeostatic conditions in the mouse. FASEB J 2008;22:3111-3119.

41 Sasaki K, Iwatsuki H, Suda M, Itano C: Accumulation and massive cell death of polymorphonuclear neutrophils in the developing bone marrow of the mouse: a histological study. Acta Anat (Basel) 1995;153: 111-118.

42 Dresch C, Flandrin G, Breton-Gorius J: Phagocytosis of neutrophil polymorphonuclears by macrophages in human bone marrow: importance in granulopoiesis. J Clin Pathol 1980;33:1110-1113.

43 Gordy C, Pua H, Sempowski GD, He YW: Regulation of steady-state neutrophil homeostasis by macrophages. Blood 2011;117:618629.

44 Szczepura KR, Ruparelia P, Solanki CK, Balan K, Newbold P, Summers C, Chilvers ER, Peters AM: Measuring whole-body neutrophil redistribution using a dedicated whole-body counter and ultra-low doses of 11lindium. Eur J Clin Invest 2011;41:77-83.

45 Stark MA, Huo Y, Burcin TL, Morris MA, Olson TS, Ley K: Phagocytosis of apoptotic neutrophils regulates granulopoiesis via IL-23 and IL-17. Immunity 2005;22:285-294.

46 Hong C, Kidani Y, A-Gonzalez N, Phung T, Ito A, Rong X, Ericson K, Mikkola H, Beaven SW, Miller LS, Shao WH, Cohen PL, Castrillo A, Tontonoz P, Bensinger SJ: Coordinate regulation of neutrophil homeostasis by liver $\mathrm{X}$ receptors in mice. J Clin Invest 2012;122:337347.
47 Mei J, Liu Y, Dai N, Hoffmann C, Hudock KM, Zhang P, Guttentag SH, Kolls JK, Oliver PM, Bushman FD, Worthen GS: CXCR2 and CXCL5 regulate the IL-17/G-CSF axis and neutrophil homeostasis in mice. J Clin Invest 2012;122:974-986.

48 Ye P, Rodriguez FH, Kanaly S, Stocking KL, Schurr J, Schwarzenberger P, Oliver P, Huang W, Zhang P, Zhang J, Shellito JE, Bagby GJ, Nelson S, Charrier K, Peschon JJ, Kolls JK: Requirement of interleukin 17 receptor signaling for lung CXC chemokine and granulocyte colony-stimulating factor expression, neutrophil recruitment, and host defense. J Exp Med 2001;194:519-527.

49 Homburg CH, de Haas M, von dem Borne $\mathrm{AE}$, Verhoeven AJ, Reutelingsperger $\mathrm{CP}$, Roos D: Human neutrophils lose their surface FC gamma RIII and acquire annexin V binding sites during apoptosis in vitro. Blood 1995;85:532-540.

50 Fadok VA, Savill JS, Haslett C, Bratton DL, Doherty DE, Campbell PA, Henson PM: Different populations of macrophages use either the vitronectin receptor or the phosphatidylserine receptor to recognize and remove apoptotic cells. J Immunol 1992;149:40294035.

51 Bratton DL, Henson PM: Neutrophil clearance: when the party is over, clean-up begins. Trends Immunol 2011;32:350-357.

52 Savill J, Hogg N, Ren Y, Haslett C: Thrombospondin cooperates with CD36 and the vitronectin receptor in macrophage recognition of neutrophils undergoing apoptosis. J Clin Invest 1992;90:1513-1522.

53 Scannell M, Flanagan MB, deStefani A, Wynne KJ, Cagney G, Godson C, Maderna P: Annexin-1 and peptide derivatives are released by apoptotic cells and stimulate phagocytosis of apoptotic neutrophils by macrophages. J Immunol 2007;178:4595-4605.

54 Perretti M, D'Acquisto F: Annexin A1 and glucocorticoids as effectors of the resolution of inflammation. Nat Rev Immunol 2009;9: $62-70$.

55 Dalli J, Jones CP, Cavalcanti DM, Farsky SH, Perretti M, Rankin SM: Annexin A1 regulates neutrophil clearance by macrophages in the mouse bone marrow. FASEB J 2012;26:387396. 